Meta

Journal des traducteurs

Translators' Journal

\title{
Un Baudelaire flamand : la traduction des Fleurs du Mal par Bert Decorte (1946)
}

\section{Spiros Macris}

Volume 62, numéro 3, décembre 2017

La traduction littéraire comme création

URI : https://id.erudit.org/iderudit/1043949ar

DOI : https://doi.org/10.7202/1043949ar

Aller au sommaire du numéro

\section{Éditeur(s)}

Les Presses de l’Université de Montréal

\section{ISSN}

0026-0452 (imprimé)

1492-1421 (numérique)

Découvrir la revue

\section{Citer cet article}

Macris, S. (2017). Un Baudelaire flamand : la traduction des Fleurs du Mal par Bert Decorte (1946). Meta, 62(3), 565-584. https://doi.org/10.7202/1043949ar

\section{Résumé de l'article}

La première traduction néerlandaise complète des Fleurs du Mal est due au poète et traducteur flamand Bert Decorte (1915-2009). Publiée en 1946, la traduction De bloemen van den booze date de la seconde moitié des années 1930 et accompagne les débuts du jeune poète. La traduction se révèle remarquable par le travail sur la forme (mètre et rime) et le rythme. L'importance accordée au mètre induit des stratégies de traduction modulées en fonction des contraintes métriques. On observe, par ailleurs, des transpositions qui relèvent de l'appropriation de sens.

Cette appropriation met en évidence l'autre versant du processus créatif à l'oeuvre dans la traduction. Celle-ci dépend du développement des ressources de la langue et de la littérature néerlandaises dans une Belgique où les francophones, qui dominaient tous les aspects de la vie économique, politique, sociale et culturelle, ont maintenu jusqu'à la fin du XIX ${ }^{\mathrm{e}}$ siècle la vision d'un pays fondamentalement monolingue. La littérature flamande doit s'imposer en tant que littérature autonome face à son influente voisine, ce qui passe d'abord par un apport flamand à la littérature française (Verhaeren, Maeterlinck, etc.), puis par la conversion d'une inspiration française, où Baudelaire joue un rôle pivot, pour lui donner une expression flamande (Van Langendonck et $K$. van de Woestijne). Les relations littéraires avec les Pays-Bas suivent une évolution similaire. La traduction des Fleurs du Mal, comme l'oeuvre poétique de Decorte, est reçue en Flandre comme le prolongement moderne de cet effort d'émancipation. La traduction doit ses traits caractéristiques à cette relation étroite d'où elle fait émerger un Baudelaire réellement flamand.
Ce document est protégé par la loi sur le droit d'auteur. L’utilisation des services d’Érudit (y compris la reproduction) est assujettie à sa politique d'utilisation que vous pouvez consulter en ligne.

https://apropos.erudit.org/fr/usagers/politique-dutilisation/ 


\title{
Un Baudelaire flamand: la traduction des Fleurs du Mal par Bert Decorte (1946)
}

\author{
SPIROS MACRIS \\ Université de Lille, Lille, France* \\ spiros.macris@univ-lille3.fr
}

\section{RÉSUMÉ}

La première traduction néerlandaise complète des Fleurs du Mal est due au poète et traducteur flamand Bert Decorte (1915-2009). Publiée en 1946, la traduction De bloemen van den booze date de la seconde moitié des années 1930 et accompagne les débuts du jeune poète. La traduction se révèle remarquable par le travail sur la forme (mètre et rime) et le rythme. L'importance accordée au mètre induit des stratégies de traduction modulées en fonction des contraintes métriques. On observe, par ailleurs, des transpositions qui relèvent de l'appropriation de sens.

Cette appropriation met en évidence l'autre versant du processus créatif à l'œuvre dans la traduction. Celle-ci dépend du développement des ressources de la langue et de la littérature néerlandaises dans une Belgique où les francophones, qui dominaient tous les aspects de la vie économique, politique, sociale et culturelle, ont maintenu jusqu'à la fin $d u x x^{e}$ siècle la vision d'un pays fondamentalement monolingue. La littérature flamande doit s'imposer en tant que littérature autonome face à son influente voisine, ce qui passe d'abord par un apport flamand à la littérature française (Verhaeren, Maeterlinck, etc.), puis par la conversion d'une inspiration française, où Baudelaire joue un rôle pivot, pour lui donner une expression flamande (Van Langendonck et K. van de Woestijne). Les relations littéraires avec les Pays-Bas suivent une évolution similaire. La traduction des Fleurs du Mal, comme l'œuvre poétique de Decorte, est reçue en Flandre comme le prolongement moderne de cet effort d'émancipation. La traduction doit ses traits caractéristiques à cette relation étroite d'où elle fait émerger un Baudelaire réellement flamand.

\section{ABSTRACT}

The first complete Dutch translation of Les Fleurs du Mal was done by the Flemish poet and translator Bert Decorte (1915-2009). Published in 1946, the translation De bloemen van den booze goes back to the second half of the 1930's when the young poet began his own writing. The translation's prime feature is the remarkable work on formal aspects (metre and rhyme) and on rhythm. The focus on meter induces different translation strategies depending on the degree of metrical constraint. Some transpositions are clearly an appropriation of meaning.

This appropriation highlights another aspect of the creative process in this translation. It depends entirely on the resources of Dutch language and literature in Belgium where the French-speaking elite dominated all aspects of social life and considered the country as monolingual French until the late nineteenth century. Flemish literature had therefore to establish itself against its influential neighbour, a process which began with a Flemish contribution to French literature (Verhaeren, Maeterlinck, etc.), then by converting a French inspiration to a true Flemish expression (Van Langendonck, K. van de Woestijne), with Baudelaire playing a pivotal role. The literary relations with the Netherlands have followed a similar evolution. The translation of Les Fleurs du Mal, as well as Decortes's own poetry, have been received in Flanders as a modern continuation of this emancipatory effort. The translation owes its features to this intimacy which engenders in turn a truly Flemish Baudelaire. 


\section{RESUMEN}

La primera traducción completa al neerlandés de Les Fleurs du Mal, publicada en 1946, es obra del poeta y traductor flamenco Bert Decorte (1915-2009). De bloemen van den booze data de la segunda mitad de la década de 1930 y pertenece al periodo inicial del joven poeta. El resultado es excelente, tanto en lo que respecta a la forma (medida y rima) como al ritmo. La importancia otorgada a la medida determina estrategias de traducción moduladas en función de las reglas métricas. Además, se observan transposiciones que corresponden a una apropiación del sentido.

Esta apropiación evidencia el otro aspecto del proceso creativo que entra en juego en esta traducción: su dependencia del desarrollo de los recursos de la lengua y la literatura neerlandesas en Bélgica, un país en el que los francófonos dominaban en esa época todos los aspectos de la vida económica, política, social y cultural, manteniendo la visión de un país fundamentalmente monolingüe hasta finales del siglo XIX. La literatura flamenca debía imponerse como literatura autónoma frente a su influyente vecina. Esto se produjo primero a través del aporte flamenco a la literatura francófona (Verhaeren, Maeterlinck, etc.) y más tarde mediante la conversión de una inspiración francesa (en la que Baudelaire desempeñó un papel esencial) a la que se le otorgó una expresión flamenca (Van Langendonck, K. van de Woestijne). Las relaciones literarias con los Países Bajos siguieron una evolución similar. La traducción de Les Fleurs du Mal y la obra poética de Decorte fueron recibidas en Flandes como una prolongación moderna de ese esfuerzo de emancipación. La traducción debe sus rasgos característicos a esa relación estrecha de la que emerge un Baudelaire resueltamente flamenco.

\section{MOTS CLÉS/KEYWORDS/PALABRAS CLAVE}

Baudelaire, métrique, néerlandais, littérature flamande, création

Baudelaire, metre, dutch, flemish literature, creation

Baudelaire, métrica, neerlandés, literatura flamenca, creación

La première traduction complète en néerlandais des Fleurs du mal paraît en 1946 sous le titre De bloemen van den booze (Baudelaire 1975, traduit par Decorte 1946) ${ }^{1}$. Son auteur est le poète et traducteur flamand Bert Decorte (1915-2009) dont l'œuvre poétique, commencée à dix-neuf ans, s'est achevée douze années plus tard avec le recueil Aards gebedenboek [Bréviaire terrien] (1947) ${ }^{2}$, l'auteur craignant de tomber dans des redites (Decorte 1971 : 187). L'essentiel de son activité littéraire est désormais tourné vers la traduction et la composition d'anthologies consacrées à des domaines poétiques peu connus.

Si la traduction domine la seconde partie de la vie de Decorte, elle est déjà présente dans la première. Lorsqu'il s'est su poète, adolescent, il se savait également traducteur et possédait déjà dans les deux domaines une certaine pratique (Decorte 1971: 74). Chaque activité nourrit l'autre, au point qu'elles se confondent parfois, unies par le même sens du rythme. Son premier poème publié est écrit, sous l'influence de Rimbaud, en une nuit. La poésie est alors devenue pour Decorte comme une symphonie qu'il porte en lui jusqu'au moment où les vers tombent en place (Decorte 1971 : 112). Le poème «De ruiters» [les cavaliers] (Decorte 1935) comporte vingt-cinq strophes de quatre hexamètres chacun, reproduisant jusque dans la disposition des rimes le schéma du «Bateau ivre». En Flandre, la critique retient le caractère vitaliste et prometteur de l'œuvre et, si l'on reconnaît une certaine parenté avec Rimbaud, c'est surtout pour regretter une composition moins rigoureuse (Vercamme 1936: 667) ${ }^{3}$. Reste qu'avec ce poème Decorte s'est fait un nom. Côté PaysBas, on voit surtout dans ces vers une imitation plutôt naïve (Du Perron 1937: 1208) . 
Mais la littérature belge néerlandophone se trouve dans une phase de reconquête et le premier recueil de Decorte, Germinal (Decorte 1937), qui porte encore la trace de ses lectures rimbaldiennes (Vermeylen 1953: 731) , est acclamé. L'écrivain André Demedts (1906-1992) voit en lui «le premier enfant prodige de notre littérature depuis Van Ostaijen. Decorte possède une vitalité exubérante qui se manifeste par des rythmes fougueux» $(1937: 520)^{6}$.

Pour la littérature flamande, la création joue sur deux plans distincts: l'accomplissement personnel, où toutes les influences sont possibles, et l'apport à une œuvre collective, une littérature encore jeune, avec ce que cela implique sur le plan culturel et politique. Tout écrivain, ou traducteur, est aussi un militant de la cause flamande. Cette dimension est beaucoup moins présente chez les critiques néerlandais qui se reposent sur une langue standardisée au $\mathrm{XVII}^{\mathrm{e}}$ siècle au sein d'une république des Provinces-Unies riche et indépendante. Dans les provinces du Sud, il faudra attendre 1830 et l'indépendance de la Belgique pour que le néerlandais puisse s'y développer de nouveau, avec une variante spécifique, créant les conditions d'une littérature nouvelle.

Aborder la traduction des Fleurs du Mal sous l'angle de la création conduit ainsi à relier le travail sur la langue, propre à l'activité de tout poète, et la constitution des moyens linguistiques et littéraires du traducteur, qui vient enrichir les moyens d'expression de cette littérature. La dialectique entre langue et parole, au sens jakobsonien, constitue la source des œuvres à venir. C'est la question du consentement dont Jean-René Ladmiral fait un préalable à toute traduction : «il n’y a de jouissance poétique, bonheur esthétique de la traduction, que si la langue est consentante» (Ladmiral 2014: 25). La notion de plaisir est centrale puisqu'elle stimule la création et favorise, en retour, l'intériorisation des règles et des structures qui en sont la condition. Dans le cadre des littératures nationales, le consentement commence par la grammaticalisation puis la standardisation de la langue. Elles associent aux motifs politiques et religieux les notions d'euphonie, d'harmonie, de rythme explorées par les poètes. Ce processus est entamé à la fin $\mathrm{du}_{\mathrm{XVI}} \mathrm{e}^{\mathrm{e}}$ siècle dans le domaine néerlandais, mais elle n'a pas bénéficié aux provinces du Sud. Il faudra attendre le poète Guido Gezelle (1830-1899) ${ }^{7}$ pour ouvrir la voie à une expression spécifiquement flamande, dans un contexte où la littérature néerlandophone est dominée par la Hollande.

Il s'agit tout d'abord d'étudier dans ces pages quelques aspects fondamentaux du processus de traduction, d'en définir les priorités et les conséquences qu'elles entraînent pour le texte. Puis l'attention sera portée sur l'origine des moyens du traducteur et la manière dont il s'insère, à travers ces moyens, dans la littérature flamande. Ces deux aspects déterminent la dimension créatrice de cette traduction.

\section{La traduction}

Bien que De bloemen van den booze soit publiée en 1946, Decorte a travaillé sur la traduction de 1935 ou 1936 à 1939 (Decorte 1971: 111; 1974: 5; 1984: 367). Elle accompagne donc la première phase de sa formation poétique. Jusqu'à 1935, il a alors vingt ans, ses poèmes sont influencés par Apollinaire et Paul Van Ostaijen. Il a quitté le lycée, supportant mal la discipline de l'enseignement catholique, et travaille dans une épicerie. Un de ses anciens professeurs, avec lequel il renoue par hasard, joue alors le rôle décisif. Il lui montre l'importance de maîtriser la versification et lui donne 
les œuvres de Rimbaud dans l'édition publiée par Paterne Berrichon en 1912. Decorte découvre une poésie qui lui est plus proche que celle de Baudelaire et, par certains égards, plus proche de ce qu'il écrit déjà (Decorte 1971: 111). L'année 1935 sera donc à la fois celle de l'irruption du rythme rimbaldien dans ses poèmes, comme le montre «De ruiters», et le début d'un travail plus réflexif et technique avec la traduction des Fleurs du mal.

Le texte utilisé pour la traduction est sans doute l'édition critique des Euvres complètes, commencée par Jacques Crépet en 1922 et achevée en 1953 par Claude Pichois, dont le volume comprenant les Fleurs du mal paraît en 1931. La traduction est présentée sous la forme d'une édition bilingue, avec le texte original à gauche et la traduction en regard, chaque poème débutant en haut de page. Le volume s'ouvre sur un avertissement dans lequel l'écrivain Herman Teirlinck (1879-1967) évoque l'expérience fusionnelle qu'il perçoit dans cette traduction et loue la sensibilité médiumnique du traducteur.

Dès les premiers vers, le lecteur comprend par quelles voies cette sensibilité s'exprime. Voici la traduction du premier quatrain de «Au lecteur»:

La sottise, l'erreur, le péché, la lésine,

Occupent nos esprits et travaillent nos corps,

Et nous alimentons nos aimables remords,

Comme les mendiants nourrissent leur vermine. (Baudelaire 1975)

Traduction de Decorte:

Zotheid en leugenleer, schraapzucht en zonde zetten

hun klauw op onzen geest en bewerken ons lijf,

en onze lieve spijt voeden we vet en stijf,

zooals de bedelaars hun ongedierte vetten.

[Sottise et erreur, lésine et péché plantent / leur griffe sur notre esprit et travaillent notre corps, / et notre cher remords nous [le] nourrissons gras et gros, / comme les mendiants engraissent leur vermine.] (Traduction de l'auteur) ${ }^{8}$

Du point de vue de la forme, la traduction suit avec précision l'original. À l'alexandrin répond l'hexamètre ïambique, le vers de référence pour la poésie néerlandaise depuis la Renaissance. C'est un vers syllabo-tonique combinant un nombre fixe de syllabes avec un nombre fixe d'accents. L'hexamètre ïambique est ainsi formé de six groupes de deux syllabes dont la seconde, c'est-à-dire les positions paires, porte l'accent. L'unité est donc le pied métrique et pas la syllabe. Le vers de Decorte est d'autant plus proche de son modèle qu'il maintient l'équivalent de la césure en plaçant en sixième position une syllabe fortement marquée suivie d'une pause. De même, la répartition des rimes masculines et féminines suit le modèle de l'original dans toutes les strophes.

Un parallélisme similaire gouverne la structure grammaticale et la distribution des unités sémantiques. Pour le premier point, la correspondance est parfaite avec notamment la séquence «, / et» au centre du quatrain (v.2 et 3 ) et le comparatif «comme» ("zooals») ouvrant le vers 3. "Péché» et «lésine» sont inversés dans le premier vers de même que «nos aimables remords» et "nous alimentons» dans le troisième vers. La correspondance entre les termes est exacte ou très proche.

Les deux occasions où la traduction s'écarte de l'original concernent des expansions. «Occupent nos esprits» devient «mettent leur griffe sur notre esprit» («zetten 
/ hun klauw op onzen geest»), ce qui demeure cohérent avec les démons (v.22), les panthères (v.29), vautours (v.30) et autres monstres (v.31). Au vers suivant, "nous alimentons» (v.3) est rendu adéquatement par "voeden we», mais le traducteur ajoute "vet en stijf» [gras et rebondi]. Il introduit ainsi une nuance reprise au vers suivant où vetten [engraisser] traduit «nourrissons", ce que l'expression "payer grassement" (v.6) de la strophe suivante ne justifie pas entièrement. La distribution du terme vet correspond presque exactement néanmoins à celui de gras dans les Fleurs du Mal.

L'explication la plus évidente de ces glissements tient aux contraintes liées à la forme. Celles-ci se révèlent fortes et, significativement, les deux passages plus libres quant au sens sont parfaitement métriques. L'analyse de la strophe montre la souplesse avec laquelle le traducteur utilise le schéma métrique.

| Zotheid | en leu|genleer, ||
hun klauw | op on|zen geest ||
| en on|ze lie|ve spijt || voeden |
| zooals | de be|delaars ||

La structure ïambique du vers - pour l'hexamètre: |w $S \mid$ w $S \mid$ w $S \mid$ w $S \mid$ w $S$ $|\mathrm{w} S|^{9}$ - joue un rôle similaire à une partition musicale. Son respect ne doit pas être mécanique sous peine de devenir insipide. Parmi les variantes les plus fréquentes, se trouve l'inversion du premier pied qui provoque un avancement du temps fort, suivi de la rencontre de deux temps faibles, puis un retour au rythme ïambique au troisième pied. Cette variante est reprise après la pause-césure, ce qui renforce à la fois le rôle de celle-ci et la musicalité du vers. Le motif est repris en écho dans la seconde moitié du vers 3 .

Chaque vers s'achève sur un pied ïambique régulier, de même que la strophe se termine par un vers ïambique régulier avec une sixième position clairement marquée, bien que ce soit l'accent secondaire du mot. Demeure cependant une zone amétrique dans le vers 2 après la pause centrale. La séquence "en bewerken" [et travaillent] avec deux temps faibles, la copule en sans autre rôle que grammatical et l'affixe inaccentué be-, tandis que l'accent sur -wérken (travailler que l'affixe rend transitif) ne peut, par sa position, être une inversion de pied valable. Il s'agit d'une zone où les contraintes de la traduction ne sont pas résolues puisque le jeu avec le mètre doit le céder au sens.

Le principal enseignement de cette strophe se vérifie sur l'ensemble de la traduction. Nous reviendrons plus en détail sur le cas, de loin le plus fréquent, du poème composé uniquement d'alexandrins pour lequel le respect du mètre est constant.

Pour les autres poèmes monométriques, le facteur décisif est la longueur du vers. Le décasyllabe est rendu par le pentamètre ïambique («Le léthé», «De lethe»), l'octosyllabe par le tétramètre ("Le vampire», "De vampier»). Mais les sept syllabes des vers de la «Chanson d'après-midi» («Namiddagliedje») deviennent également des tétramètres ïambiques, tout en respectant la distribution des rimes masculines et féminines. Le maintien de la mesure ïambique a été décisif pour trouver l'équivalent métrique du seul poème heptasyllabique des Fleurs du Mal.

On observe en effet le même choix dans le poème hétérométrique «Le poison» («Het vergift»). Les quatre strophes de cinq vers sont composées d'alexandrins (vers 1,3 et 5) et d'heptasyllabes (vers 2 et 4 ), ce qui est également un cas unique. Tout en respectant la disposition de la strophe, le jeu des rimes et l'alternance entre les rimes masculines et féminines, Decorte remplace, là aussi, les heptasyllabes par des tétramètres ïambiques. La transposition d'un vers impair, à la coupe toujours inégale, par 
un vers doublement pair, deux groupes de deux pieds de deux syllabes, paraît fautive. Il faut cependant raisonner en nombre d'accents et non de syllabes, c'est déjà ainsi qu'au XIII ${ }^{\mathrm{e}}$ siècle Hadewijch d'Anvers transposait les vers de Moniot d'Arras (Grijp 1992). Il s'agit ici de contrefacture et donc de chant, mais la dimension vocale, physique, est indissociable de la métrique accentuelle. À la même époque, Willem van Afflighem (1210-1297) écrit la Leven van Sinte Lutgard [Vie de sainte Lutgard] dont les 20406 vers qui nous sont parvenus se présentent comme des octosyllabes réguliers généralement interprétés comme des tétramètres ïambiques (Zonneveld 1992). Il existe une proximité entre les deux types de versification, du moins sur le plan linguistique car le poème de Willem ne présente pas cette intégration entre le mètre et la langue propre au vers développé à la Renaissance. L'alternance accentuelle n'est qu'un aspect des formes métriques.

Plusieurs poèmes reposent sur des strophes composées de deux mètres différents, une répétition souvent associée à la reprise d'un vers ou d'un demi-vers. Les combinaisons d'alexandrins et d'octosyllabes sont rendues par des vers de même longueur, avec la même alternance des rimes masculines et féminines. Il en va de même pour le sonnet «La musique» («Muziek») qui est le seul à mêler alexandrins et pentasyllabes.

Le poète maintient son exigence dans des compositions basées sur des vers courts, mais une analyse détaillée montre que les vers de 4 ou 5 syllabes deviennent irréguliers, et tendent à gagner une syllabe («L'amour et le crâne», «L’invitation au voyage», «Le jet d'eau»).

Afin de densifier la charge sémantique, le traducteur exploite la capacité du néerlandais à combiner des mots pour en créer de nouveaux, comportant généralement une alternance accentuelle (Neijt et Schreuder 2007). Ainsi dans le refrain de «L'invitation au voyage» («Heimwee» [Nostalgie]), " schoonheidsrust» associe rust [calme, repos, équilibre] à schoonheid [beauté] pour traduire "ordre et beauté». Pareillement, zinnelust [volupté, désir des sens] porte un accent primaire sur la première syllabe et un accent secondaire sur la troisième.

Là, tout n'est qu'ordre et beauté,

Luxe, calme et volupté. (Baudelaire 1975)

Daar is alles schoonheidsrust, $\quad \mathrm{S} w \mathrm{~S} w \mathrm{Sw} \mathrm{S}$

weelde, stilte en zinnelust. $\quad \mathrm{S}$ w $\mathrm{S}$ w w $\mathrm{S}$ w $\mathrm{S}$

[Là, tout n'est que repos de beauté, / abondance, calme et désir des sens.]

Le premier vers, avec ses sept syllabes, sera lu comme vers trochaïque incomplet. Le second vers présenterait le même profil métrique s'il n'y avait la copule en. Ce temps faible réintroduit une conclusion ïambique, renforce l'accent secondaire sur zínnelùst, ce qui équilibre par une clausule le refrain. La combinaison 7 syll. +8 syll. rend ainsi compte à travers le rythme de la tripartition $2+2+3$ de l'original qui a même valeur iconique avec l'équilibre entre «luxe», «calme» et «volupté».

Pour l'ensemble des 42 vers du poème, la transposition donne des résultats similaires. La longueur de 17 pentasyllabes sur 24 et 6 heptasyllabes sur 18 est maintenue. La relative stabilité du pentasyllabe se retrouve parmi les 19 vers restants où les 7 vers de cinq syllabes en gagnent une tandis que douze heptasyllabes sont rendus le plus souvent par des octosyllabes et une fois par un décasyllabe; dans les trois cas où il y a une réduction, la traduction revient deux fois à six syllabes et une seule fois, au 
vers 23, à un vers impair de cinq syllabes. Mais même ce dernier exemple confirme la tendance à compléter le mètre en revenant à un vers pair. Il s'agit en l'occurrence d'un vers trochaïque avec une rime féminine: «Oosterpracht en -pralen» (v.23 «La splendeur orientale») qui comporte trois pieds ( $\left.\left|S_{w}\right| S_{w}\left|S_{w}\right|\right)$, rendant métrique la finale non accentuée.

Une analyse systématique des 27 premiers sonnets en alexandrins, ce qui représente plus de la moitié des 47 sonnets de ce type que comptent les Fleurs du mal, montre le travail du traducteur sur les vers et leur harmonie. Ces résultats sont représentatifs, car ils confirment les valeurs obtenues avec la moitié de cette population.

L'analyse a consisté à définir les syllabes métriques puis à leur attribuer une valeur de 1 à 4 selon l'intensité de l'accentuation. Le but est de dépasser l'opposition binaire accentué / non accentué, afin de tenir compte des effets de hiérarchisation dans les mots composés, les rencontres d'accents et d'inclure l'accent métrique, notamment pour les monosyllabes. Il s'agit donc de mettre en valeur le modèle métrique implicite et d'éclairer la manière dont il est réalisé. Dans le contexte de la traduction, cette réalisation est étroitement liée à la manière dont Decorte gère des impératifs contradictoires du contenu et de la forme. La distinction reste pertinente pour analyser le processus et apparaît, par exemple, dans les traductions pseudo régulières de poèmes réguliers. Elle disparaît lorsque les deux se renforcent mutuellement et trouvent leur équilibre dans une même unité.

Le néerlandais possède un registre de marquage étendu. Johannes Kinker distingue ainsi, dans sa Prosodia (1810; Macris 2016), vingt-sept longueurs différentes, de la syllabe atone consonne + schwa à la voyelle longue entourée de deux amas de consonnes avec un [r] comme 't vreemdst [le plus étrange] regroupées en cinq classes. Bien que centrée sur la durée, cette systématisation est proche de la notion actuelle de poids des syllabes. Cette approche demeure intéressante parce que son étude de la prosodie est faite dans une perspective métrique. Il s'agit en effet d'évaluer dans quelle mesure les mètres grecs et latins peuvent enrichir la poésie néerlandaise. Les quatre valeurs retenues ici offrent un système simple et lisible qui tient compte des différents types d'accents:

1: Syllabes légères $(\mathrm{CV}, \mathrm{VC})$ non accentuées;

2: Syllabes intermédiaires (CVV, CVC...) non accentuées ou syllabes légères accentuées;

3: Syllabes intermédiaires et syllabes lourdes (CVVC, CCVVC...) accentuées;

4: Syllabes superlourdes accentuées (CCVVCCC...).

La distribution des syllabes n'est pas arbitraire dans le schéma métrique, poids et charge sémantique allant souvent de pair. L'accent de mot dépend d'une part du poids de la syllabe et d'autre part de la position, déterminée par le premier pied trochaïque constitué à partir de la droite du mot (Kooij et Van Oostendorp 2003: 105128; Booij 1995: 96-124). La plupart des syllabes lourdes ou superlourdes portent un accent de mot et occupent une position clé à la fin du vers ou, plus rarement, à la césure. Le traducteur leur prête donc une attention particulière.

Le tableau ci-dessous résume deux types de données par sonnet: la moyenne de la valeur accentuelle par position, avec la différence entre les deux syllabes composant le pied, et l'écart type qui indique si la moyenne résulte de résultats disparates ou non. 
TABLEAU 1

Données regroupées par sonnet

\begin{tabular}{|l|c|c|c|c|c|c|c|c|c|c|c|c|}
\hline Pieds & \multicolumn{2}{|c|}{ P1 } & \multicolumn{2}{c|}{ P2 } & \multicolumn{2}{c|}{ P3 } & \multicolumn{2}{c|}{ P4 } & \multicolumn{2}{c|}{ P5 } & \multicolumn{2}{c|}{ P6 } \\
\hline Positions & $\mathbf{1}$ & $\mathbf{2}$ & $\mathbf{3}$ & $\mathbf{4}$ & $\mathbf{5}$ & $\mathbf{6}$ & $\mathbf{7}$ & $\mathbf{8}$ & $\mathbf{9}$ & $\mathbf{1 0}$ & $\mathbf{1 1}$ & $\mathbf{1 2}$ \\
\hline Moyennes & 24,78 & 35,04 & 19,56 & 37,59 & 17,7 & 38,48 & 23,3 & 36,33 & 19,48 & 37,93 & $\underline{17,41}$ & $\underline{43,07}$ \\
\hline Différences & \multicolumn{2}{|c|}{10,26} & \multicolumn{2}{|c|}{18,04} & \multicolumn{2}{|c|}{20,78} & \multicolumn{2}{|c|}{13,04} & \multicolumn{2}{|c|}{18,44} & \multicolumn{2}{|c|}{25,67} \\
\hline Écarts types & 2,76 & 2,97 & 2,95 & 2,63 & 2,4 & 2,52 & 3,17 & 3,51 & 2,28 & 2,77 & 1,77 & 2,04 \\
\hline
\end{tabular}

Le premier enseignement est la position éminente du sixième pied (P6). Il cumule à la fois, et de loin, l'intensité maximale sur sa position forte et l'intensité minimale sur sa position faible, présentant ainsi le contraste le plus fort entre les deux positions constitutives du pied.

Le $\mathrm{P} 3$ se distingue aussi nettement, mais avec une prééminence moins forte et un contraste plus faible entre les deux positions. Decorte tient donc manifestement compte de la césure dans sa transposition métrique alors qu'elle ne joue pas un rôle structurel dans l'hexamètre originel, celui de Joost van den Vondel (1587-1679), par exemple. Cette évolution apparaît à la fin du XVII siècle, sous l'influence du classicisme.

La mesure de l'intensité met également en évidence la cohérence de l'ordre qui régit les pieds restants. $\mathrm{P} 5$ précède en intensité $\mathrm{P} 2$ si bien qu'une pente se dessine dont le point bas se trouve en $\mathrm{P} 1$ et $\mathrm{P} 4$. Bien qu'il ne s'agisse pas de véritables hémistiches, deux groupes émergent avec une progression régulière en contraste et en intensité, mais avec un léger décalage. La série majeure est constituée par la seconde moitié du vers avec des contrastes de 13,04, 18,44 et 25,67; la série mineure, les pieds 1 à 3 , présentant des contrastes de 10,26, 18,04 et 20,78.

La mesure de l'écart type complète ces données. Elle renforce tout d'abord la prééminence du dernier pied puisque ses deux positions réunissent les écarts types les plus faibles. La donnée moyenne résulte donc de chiffres présentant une faible dispersion. Le P6 se révèle ainsi particulièrement stable. À l'opposé, la situation P4 réunit les deux écarts types les plus forts. Une deuxième zone instable apparaît aux positions 2 et 3, c'est-à-dire à cheval sur P1 et P2.

La mesure d'intensité confirme le sentiment du lecteur, les vers de cette traduction sont de véritables vers et non le résultat d'une simple arithmétique. Elle met aussi en évidence les zones privilégiées par le traducteur, où la cohérence est la plus forte et la contrainte formelle s'est révélée la plus créatrice. Il s'agit des P6 et P3 avec les P5 et $\mathrm{P} 2$ qui les précèdent. Les tensions de la traduction se trouvent alors reportées vers les $\mathrm{P} 1$ et $\mathrm{P} 4$, surtout le $\mathrm{P} 4$ où la variance est la plus grande. La dispersion des données pour le $\mathrm{P} 1$, pourtant bien inférieure à celles du P4, s'explique par l'inversion fréquente du premier pied. Elle n'est donc pas liée au processus de traduction. Cela explique le report de la zone de tension vers les positions 2 et 3 .

La position P4 se trouve prise entre deux zones stables. Un des traits qui manifestent la rigidité de la fin des vers est la fréquence des mots longs. Ceux-ci épousent le mètre par le jeu des accents primaires et secondaires ou la réduction du schwa. La combinaison de termes, très productive en néerlandais, évite les mots-outils. On trouve ainsi, sur l'ensemble du recueil, $70 \%$ des mots de plus de 18 caractères en fin de vers ( 9 sur 13). Cette proportion diminue si l'on baisse le seuil à 17 caractères, mais reste significative: $58 \%$ des mots se trouvent en fin de vers et $10 \%$ au début, où ils 
jouent un rôle similaire. Seuls $30 \%$ des mots sont à une syllabe au moins du bord du vers. L'exemple des vers 15 à 19 du «Spleen. J'ai plus de souvenirs...», montre l'utilisation que fait le traducteur des mots longs:

1 Rien n'égale en longueur | les boiteuses journées,

2 Quand sous les lourds flocons | des neigeuses années

3 L'Ennui, fruit de la mor|ne incuriosité,

4 Prend les proportions | de l'immortalité. (Baudelaire 1975)

1 Niets kan de manke duur | der dagen evenaren,

2 wen, onder't zware dons | der sneeuwbeladen jaren,

3 verveling, vrucht der som|bre onwetensgierigheid,

4 verhoudingen aanneemt | van onverganklijkheid.

[Rien ne peut la durée boiteuse des jours égaler, / lorsque, sous le lourd duvet des années chargées de neige / l'ennui, fruit de la morne (sombre) incuriosité, / prend les proportions de l'impérissabilité.]

Outre le respect des contraintes formelles, on observe un grand parallélisme dans la traduction. Le seul déplacement se situe au premier vers où «manke» [boiteux] porte sur la durée de la journée («de duur der dagen») et non sur la journée ellemême. "Duur» offre un appui solide pour la césure tandis que «evenaren " [égaler] assure une rime riche avec le vers suivant, comme l'original. La séquence «der dagen evenaren " ouvre aussi une série d'assonances et de rimes intérieures au vers suivant: «zware» [lourd], «sneeuwbeladen jaren» [années chargées de neige].

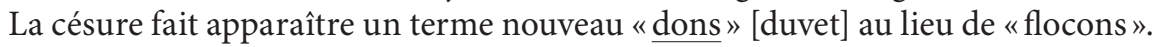
Quoique satisfaisante du point métrique, l'image "'t zware dons" [le lourd duvet] paraît plus contradictoire que "lourds flocons", tout en allant dans le même sens. L'idée de poids est reprise avec le terme "sweeuwbeladen" [chargées de neige] qui prolonge ainsi «lourds». À partir de là, le parallélisme entre l'original et la traduction est presque parfait.

Le vers 3 répond terme à terme au vers français. La traduction de «morne» par le terme néerlandais, emprunté au français, somber, ici utilisé avec la réduction de la syllabe atone de la forme accordée sombere $>$ sombre, demeure très proche. Le recours au composé "onwetensgierigheid", qui constitue le demi-vers, est servi par «incuriosité». Mais la traduction de curiosité est nieuwsgierigheid (nieuw $=$ nouveau), alors que wetensgierig, rare mais transparent, signifie avoir soif de connaissances. La construction onwetensgierigheid insiste sur l'absence d'intérêt pour le monde, ce qui rejoint l'incuriosité par une autre voie, bien moins courante mais métrique.

Le quatrième vers se révèle encore plus proche de l'original et le seul changement est dû à la syntaxe qui oblige à placer le verbe après le complément, même s'il s'intercale dans le groupe nominal. Le terme «immortalité», à la fin du vers, n'est pas rendu par onsterfelijkheid, mais par onvergankelijkheid [impérissabilité], ce qui sécularise quelque peu l'image. Par une réduction du schwa, Decorte rétablit l'alternance accentuelle, avec une rime masculine, de manière à renforcer mètre et rime par un sens plein: van ònvergánkelijkheìd > «|| van òn|vergán|klijikheìd |».

Ce renforcement crée une difficulté autour de la césure. Dans le vers 3, elle se manifeste par la double réduction dont fait l'objet sombre avec la suppression du schwa et la liaison avec le mot suivant, ce qui suit une logique française basée sur le $e$ muet alors que le schwa néerlandais occupe toujours une position s'il est maintenu: sómbere 
onwétensgìerighèid donne la suite mesurée : «der sóm || br’onwé|tensgìe|righèid». De la même manière, la césure du vers 4 suit une syllabe lourde mais non accentuée: "verhóu|dingen | áanneemt || van ón|vergàn|klijkhèid», où l'on trouve également une position 4 non accentuée.

Popularisée par les poètes novateurs de la génération de 1880, la création de mots nouveaux, donc de sens nouveaux, par la combinaison de deux termes connus ouvre un espace plus grand à l'imagination. La traduction en tire parti en déplaçant insensiblement les repères du texte. Les exemples ne sont pas rares: "verrukkingsdroesem» dans la sixième strophe de «Namiddagliedje» ("Chanson d'après-midi»). Les composés en sont: verrukking, qui signifie ravissement, au sens fort, extase, jouissance et

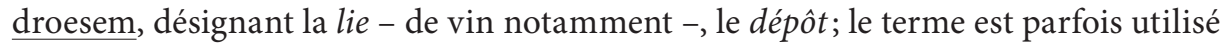
au sens de diable, par confusion avec droes dont ce sens est attesté. La proximité de $\underline{\text { roes, }}$ ivresse, joue également. La connexion par juxtaposition est assez lâche pour permettre plusieurs lectures.

1 Tes hanches sont amoureuses

2 De ton dos et de tes seins,

3 Et tu ravis les coussins

4 Par tes poses langoureuses. (Baudelaire 1975)

1 Uw heupen buigen naar uw boezem

2 en rug verliefd omhoog, en gij

3 drenkt door uw smachtend lijfsgevlei

4 de kussens met verrukkingsdroesem.

[Vos hanches se penchent (se plient) vers votre poitrine / et dos (,) amoureusement dressées, et vous / abreuvez par votre cajolerie charnelle désirante / les coussins de la trace (l'ivresse) d'extase.]

Le passage de l'heptasyllabe au tétramètre ïambique donne plus d'espace à la traduction. Celle-ci devient plus explicite et donne à la sensualité de l'original un tour plus concret: «sont amoureuses» devient «se plient vers votre poitrine et dos», "par tes poses langoureuses» prend une forme plus concrète avec «les cajoleries du corps pleines de désirs» et "tu ravis les coussins» se transforme en une image dont le caractère composite ne la rend pas moins précise: «vous imprégnez des coussins d'une lie de jouissance / ivresse de l'extase». Si cette interprétation n'est pas absente chez Baudelaire, son émergence si nette dans la traduction doit certainement autant à Rimbaud qu'à l'inspiration parfois très sensuelle du premier recueil du poète-traducteur lui-même. Ces influences, qui plongent aussi leurs racines dans le terreau des Fleurs du Mal, en prolongent l'écho à travers la traduction.

\section{Langue et littérature}

Cette dimension historique, collective, trouve une expression particulière dans la traduction de Decorte et influe sur le processus créatif. Peter Verstegen, traducteur de l'une des deux versions des Fleurs du Mal publiées aux Pays-Bas en 1995, considère que la langue de Decorte est un «idiome qui ne peut être considéré comme un néerlandais régulier» (Verstegen 1995$)^{10}$.

En réalité, la distance est linguistique et littéraire. Le premier aspect, déjà évoqué, tient au rôle central des Provinces-Unies dans la standardisation du néerlandais au 
début du $\mathrm{XVII}^{\mathrm{e}}$ siècle. Ce processus a lieu dans un contexte dominé par le calvinisme et passe notamment par une traduction ad fontes de la Bible, traduction dont la réalisation et, surtout, la diffusion sera appuyée par les États Généraux des Provinces Unies. Dans le même temps, la contre-réforme transforme profondément les provinces méridionales demeurées sous autorité espagnole. Coupé de la vie intellectuelle et littéraire des Provinces-Unies, écarté des centres du pouvoir, le néerlandais n’a rien à opposer à l'influence du français au XVIII siècle et moins encore à la bourgeoisie entrepreneuriale francophone dont le développement est stimulé par la politique économique autrichienne, puis l'annexion par la France durant deux décennies.

Le renouveau littéraire aux Pays-Bas est porté par la génération dite de 1880 qui domine les débats à la fin du siècle et oriente encore la réflexion sur la littérature au début $\mathrm{du} \mathrm{xx}^{\mathrm{e}}$ siècle. L'influence principale est anglo-saxonne (Shelley, Keats, Wordsworth, Leigh Hunt) et allemande (Von Platen). Les prosateurs se tournent vers Zola et les naturalistes français pour rompre le carcan d'une littérature mise au service de la morale calviniste. Pour tous, la sensation est la source de la création, et l'expérience de la beauté remplace l'expérience du divin. Le pentamètre ïambique devient le vers de référence. Il offre une grande souplesse rythmique, parfois exploitée à l'extrême comme peut le faire Herman Gorter (1864-1927) dans Mei [Mai] (1889). Dans cette épopée lyrique de 4381 vers, le poète renforce les enjambements au point de faire parfois tomber la fin d'un vers au milieu d'un mot. La poursuite de l'immédiateté de la sensation le pousse à une expérience radicale de déconstruction du vers et des structures grammaticales (Verzen [Vers], 1890).

L'œuvre de Baudelaire s'oppose point par point à ce programme. La génération de 1880 ne s'est pas intéressée à son œuvre (Halsema 1995), malgré les efforts d'un Frans Erens (1857-1935). Celui-ci a écrit, en 1883, un long article sur le poète (repris dans Erens 1906, trad. Macris 1988) et a défendu à Amsterdam la jeune poésie française qu'il connaissait bien pour avoir vécu à Paris de 1880 à 1883 où il a fréquenté les groupes décadents et s'est lié d'amitié avec Jean Moréas et Maurice Rollinat. Les traductions des Fleurs du Mal demeurent sporadiques. La seule exception notable est due au poète, traducteur et théoricien Albert Verwey (1865-1937). L'exception est cependant tardive par rapport au mouvement de 1880, puisque l'ensemble de ses traductions baudelairiennes, dix-neuf en tout, paraît dans la revue De beweging [Le mouvement] en 1914 et 1919.

Decorte a peut-être lu ces traductions, mais, s'il en a tenu compte, c'est pour s'en éloigner. Verwey a pourtant opté, lui aussi, pour une traduction en vers réguliers, sauf pour des mètres courts comme "L'amour et le crâne» où la traduction devient irrégulière. Il respecte également les rimes et l'alternance de finales masculines et féminines. Les différences sont cependant bien plus importantes. Pour les quatre sonnets traduits en hexamètres ( La beauté», «Semper aedem», «La cloche fêlée» et «La mort des pauvres»), les données chiffrées mettent en évidence la structure ïambique du vers et le même poids éminent de la dernière position métrique, mais avec une variation plus importante. La position 6 n'est en revanche pas marquée et arrive en sixième place dans l'ordre d'intensité, avec un écart type double de celui de Decorte. L'hexamètre de Verwey n'est donc pas de type alexandrin.

Plus encore, Verwey choisit de modifier le type de vers dans la majorité de ses traductions en remplaçant l'alexandrin par un pentamètre ïambique, soit onze poèmes sur dix-huit (excluant «L'amour et le crâne»). Exprimé en nombre de vers 
pour tenir compte de la longueur des poèmes, le passage de douze à dix syllabes concerne les trois quarts des occurrences. Cette décision trouve sa source dans la poétique appliquée par Verwey à la traduction. La traduction est pour lui le prolongement de la lecture. Une lecture si précise et approfondie que le poète ne peut s'empêcher de retrouver l'original avec ses propres mots, dans sa propre langue. Il cherche le lien fondamental qui fonde l'unité de la forme et du sens - un des principes esthétiques fondamentaux du mouvement - pour retrouver la musicalité de l'original. Verwey insiste souvent, comme le montre Martin Hietbrink (1999) ${ }^{11}$, sur la nécessité de respecter strictement la forme de l'original et l'a mis en pratique dans sa traduction de Dante. Le thème du traducteur-poète domine au cours du premier tiers du $\mathrm{XX}^{\mathrm{e}}$ siècle, notamment dans les débats autour des traductions de Shelley (Naaijkens 2002). La liberté du poète, sa fidélité supérieure à l'esthétique qui ancre le créateur dans sa langue et son époque l'emporte donc. Verwey, et sa génération, ont fait du pentamètre le moyen de leur libération créatrice, s'opposant doublement à l'influence française: d'abord en rompant avec la transformation progressive de l'hexamètre en alexandrin sous l'influence du classicisme à la fin du XVII ${ }^{\mathrm{e}}$ siècle, puis en privilégiant les liens avec la poésie anglaise. Dans un article publié en 1907 dans le Mercure de France, Verwey présente ainsi le mouvement dont il est l'un des fondateurs:

Pour Kloos la poésie était dans le sentiment. Pour moi, dans l'imagination. Mais, pour tous, elle existait en dehors du raisonnement. Nos formes étaient les sonnets, les vers libres, les ïambes à cinq pieds, avec et sans rimes, en sorte que le sonnet n'était qu'en vers de cinq pieds, et que l'alexandrin était entièrement banni de nos poèmes.

Perk et Kloos avaient lu les poètes allemands et anglais. Au moment de la fondation de la revue, Keats et Shelley avaient, chez nous, la plus grande influence. Des poètes français, nous n'en aimions alors pas un seul. En Hugo, nous ne voyions rien qu'une sorte de rhétorique plus colorée. Verlaine ne nous fut connu que bien plus tard. (Verwey 1907: 39-40)

Il rationalisera plus tard cette orientation en la plaçant dans le cadre d'une opposition plus ou moins consciente entre une tendance à la régularité apportée par l'influence romane et la propension, dans le Nord, à rompre cette régularité par un mouvement accentuel (voir Verwey 1931: 44). Les traductions de Baudelaire se trouvent ainsi prises entre deux poétiques et Verwey ne les fera pas figurer dans l'anthologie de ses traductions, Poëzie in Europa (1920).

La comparaison entre des traductions en pentamètres et en hexamètres de type alexandrin met en évidence le rôle de la césure dans ce qui est en réalité un mètre composé, chaque partie ayant son indépendance. Le pentamètre n'interdit pas cet équilibre, mais le rend incertain, dépendant de la lecture. Ainsi, dans les premiers vers de «Je n’ai pas oublié, voisine de la ville», Verwey suit, comme Decorte, le découpage des hémistiches qui constitue des unités sémantiques simples:

Je n'ai pas oublié, voisine de la ville,

Notre blanche maison, petite mais tranquille; (Baudelaire 1975)

La traduction de Verwey:

Ik denk nog dikwijls aan ons witte huis,

Niet ver van stad, klein maar zonder gedruis;

[Je pense souvent encore, à notre maison blanche /Non loin de la ville, petite mais sans agitation;] 


\section{Et Decorte:}

Ons witte huis, dat klein maar rustig was gezeten

nabij de stede, heb ik nooit of nooit vergeten,

[Notre maison blanche, qui était installée petite mais au calme / près de la ville, je ne l'ai jamais oubliée,]

Mais si la grammaire n'appuie pas le mètre, le pentamètre néerlandais retrouve ce mouvement qui en fait l'unité. L'effet d'enjambement à la césure, comme dans le vers 7 du même poème («Semblait, grand œil ouvert / dans le ciel curieux,»), est alors perdu. La souplesse du vers, recherchée par Verwey et ceux de sa génération, le prive d'un certain nombre de moyens lorsqu'il s'agit de traduire une poésie pour laquelle la contrainte formelle joue un rôle important. Ce rapport serait peut-être différent avec l'alexandrin de Verlaine, par exemple, car Baudelaire maintient un alexandrin classique. Cependant, même s'il introduit, dès la première publication des Fleurs du mal, des variations dans le schéma classique, la structure de base du vers majeur français demeure active, même sous une forme imparfaite ou détournée, comme le montre l'œuvre d'Yves Bonnefoy (Gouvard 2000; 2007).

Le sacrifice de deux syllabes entraîne parfois des contractions de sens ou des impasses. Dans «Je n’ai pas oublié, voisine de la ville», le premier cas est illustré par le vers 3 : «Sa Pomone de plâtre et sa vieille Vénus» rendu par Verwey: «Zijn Venus en Pomona, wit en koud» [Ses Venus et Pomone, blanches et froides] où «de plâtre» reste perceptible dans «wit » alors que «koud» peine à rendre «vieille». Decorte tend à aller au-delà de l'original: «de plaasteren Pomone en 't oude Venusbeeld ». Le terme «plaasteren" est propre au néerlandais du Sud (comme "nooit of nooit» dans le vers précédent) et il surtraduit «Vénus» par «Venusbeeld» [statue de Vénus]. Mais l'opposition des deux mètres n'est pas si claire.

Ainsi, la dernière strophe de la «muse malade»:

Comme les sons nombreux des syllabes antiques,

Où règnent tour à tour le père des chansons,

Phœbus, et le grand Pan, le seigneur des moissons. (Baudelaire 1975)

Verwey interprète correctement «les sons nombreux des syllabes antiques » qu'il rend, en l'explicitant, par: "Zooals 't antieke woord zijn rijkdom toomde / Door tal en maat» [Comme le mot antique maîtrisait sa richesse / par le nombre et la mesure], ce qui occupe presque la moitié du tercet. La présentation des dieux est légèrement condensée: «le père des chansons, / Phœbus» devient "Phœbus, le seigneur du Chant»; et "le grand Pan, le seigneur des moissons» donne «Pan le dieu de la Moisson ». Leur règne alterne, mais Verwey attribue la première place à Pan, sans que cela apparaisse comme une intervention:

Zooals 't antieke woord zijn rijkdom toomde

Door tal en maat, zoodat Pan de Oogstgod eerst

En dan ook Phoebus, heer van 't Lied, er heerscht.

[Comme le mot antique maîtrise sa richesse / par le nombre et la mesure, si bien que Pan le dieu de la Moisson d'abord / et puis aussi Phobus, le seigneur du Chant, y règnent.]

Plus exubérant que précis, Decorte prend «nombreux» au sens de «sons multiples» qui mènent une sarabande. Il distribue des éléments selon l'original, qu'il suit de près, mais augmente «chanson» par un ajout «des luths»: 
lijk de veelvoud'ge klank der oude silben stoeide, waarin om beurten heerscht Phoibos, de vader van het lied der luiten, en de vorst der velden, Pan.

[comme le son multiple des anciennes syllabes s'ébattait, / dans lequel tour à tour règnent Phœbus, le père / du chant des luths, et le seigneur des champs, Pan.]

Cette tendance se vérifie sur l'ensemble du poème. Verwey tend vers une traduction plus synthétique, quitte à éliminer certaines nuances et donner une nuance prosaïque («muse» devient «zangster» [chanteuse] dans le titre). Le vers 7, «Le cauchemar, d'un poing despotique et mutin» devient «De droomgeest, wild van vuist, voor rede blind " [L'esprit du rêve, le poing sauvage, aveugle à la raison], où les déterminants sont rendus de manière approximative. Dans «Je voudrais qu'exhalant l'odeur de la santé» (v.9), sujet et verbe disparaissent même complètement. À l'opposé, Decorte amplifie et préfère parfois interpréter. Là où le poète voit «s'étaler sur ton teint / La folie et l'horreur, froides et taciturnes», le traducteur découvre «uw tint bewolkt / door waanzin zwijgend in een kleed van angst geborgen" [votre teint ennuagé / par la folie muette enveloppée dans un vêtement d'angoisse].

Le choix du mètre pour la traduction de la poésie régulière repose davantage sur l'ancrage historique et littéraire du traducteur que sur un principe d'équivalence horizontale, de forme à forme. Le succès des traductions allemandes de Baudelaire par Stefan George (1868-1933), un ami de Verwey, va dans le même sens. George cherche des solutions de rechange à l'alexandrin et a majoritairement recours au pentamètre ïambique. Verwey est un des artisans de la libération du vers néerlandais de l'influence française. Pour Decorte ce rapport est au contraire naturel, tout comme son affinité avec Baudelaire puis Rimbaud: son premier recueil, Germinal (1937), est écrit très majoritairement en hexamètres généralement césurés. Les réactions opposées des critiques hollandais et flamands, évoquées au début de cet article, montrent que les différences entre les deux traductions révèlent les différences de fond entre les deux littératures (De Geest 2012; Brems 2012; voir Decorte 1937/2012: vol. 2, 133-184).

La comparaison entre ces traductions montre aussi que le choix du mètre ne doit pas être isolé des autres aspects de la traduction. Le jeu des accents et des réductions de voyelles atones rend le médium linguistique assez souple, ce dont les traducteurs donnent involontairement une illustration. Ils traduisent avec les mêmes mots, suivant le français pas à pas, le vers «Avec une indicible et mâle volupté » ("Élévation ", v.8), mais le résultat tient, dans un cas, en dix syllabes et, dans l'autre, en douze: «Met / on/uit/spreek/bre / en / man/lij/ke / lust» (trad. Verwey); «met / on/uit/spreek/ba/ ren / en / man/ne/lij/ken / lust» (trad. Decorte).

Par rapport à Verwey, Decorte se distingue par le recours à des formes régionales, caractéristiques du néerlandais du Sud ou archaïsantes et, de manière plus caractéristique, la longueur des mots. Pour les mêmes titres, il utilise un nombre supérieur de plus de la moitié de termes avec quatre ou cinq syllabes (107 contre 68 pour Verwey). L'observation vaut également pour les traductions néerlandaises plus récentes. La comparaison montre que la force de la traduction de Decorte est de recréer un univers cohérent et personnel, puisé dans un particularisme culturel et linguistique en pleine affirmation et dont il exalte la force expressive. Côté hollandais, la traduction ne se déploie pas parce que cet univers poétique est étranger à ce que représente Baudelaire à la fin du siècle. Seules quelques figures isolées feront excep- 
tion. Les traductions de Verwey portent la trace de dissonances poétiques que la comparaison révèle.

Decorte s'attache davantage à une langue littéraire parfois plus ancienne, mais sans exclusive. Ce goût pour une langue plus ancienne explique, par exemple, la traduction du poème latin «Franciscæ meæ laudes» en moyen néerlandais. Il utilise des mots rares comme scharnier (sens moderne: charnière) dans le sens de charnier, mais ponctuellement. La véritable différence réside dans les images et leur registre. Ainsi, lorsque dans le «Châtiment de l'orgueil» («De hoogmoed gestraft») un «homme monté trop haut» s'écrie, «transporté d'un orgueil satanique»:

« Jésus, petit Jésus! je t’ai poussé bien haut!

Mais, si j'avais voulu t'attaquer au défaut

De l'armure, ta honte égalerait ta gloire, (Baudelaire 1975)

Decorte traduit:

- „Ei Jezus, keutelkind, 'k heb je wel hoog gestuwd,

maar had ik voor de grap een stok in 't wiel geduwd,

dan zou je schande vast je glorie evenaren,

[Eh Jésus, petit biquet, je t’ai bien poussé haut, / mais si je t'avais, pour plaisanter, poussé un bâton dans la roue, / alors ta honte aurait certainement égalé ta gloire.]

L'adresse, en souligné simple, introduit un glissement de ton. L'exclamation Ei, qui remonte au moyen néerlandais, s'accorde à l'expression familière et régionale keutelkind, composé de keutel, signifiant petite crotte (de lapin par exemple), et kind (enfant). Ce caractère familier est prolongé par la substitution de l'image attaquer au défaut de l'armure, qui conserve une trace de noblesse, par une image populaire: «maar ik had voor de grap een stok in 't wiel geduwd" [mais j'avais poussé, pour plaisanter, un bâton dans la roue].

«Le vin des chiffonniers» rencontre cet esprit flamand plus concret, ce qui se signale par une musicalité plus riche de la traduction où les sons se répondent, portés par le rythme ïambique. On voit ainsi au vers 18, «l'énorme Paris» traduit de manière imagée et adéquate par «den draak Parijs» [le dragon Paris] qui conserve la part essentielle d'énorme qu'une traduction plus conventionnelle aurait réduit à une question de taille. De plus, draak renforce la cohésion du vers tant par le sens, le dragon baudelairien vomit «un tas de débris» au lieu de flammes, que par l'assonance draak à la césure et uitgebraakt [vomi] à la rime. Le vers 16 «Vomissement confus de l'énorme Paris», donne: «die vormloos door den draak Parijs werd uitgebraakt» [qui informe par le dragon Paris fut vomi].

Dans «Le vin de l'assassin» on constate le même type d'appropriation, dès la première strophe:

Ma femme est morte, je suis libre!

Je puis donc boire tout mon soûl.

Lorsque je rentrais sans un sou,

Ses cris me déchiraient la fibre. (Baudelaire 1975)

Mijn wijf is dood, ik ben verrezen!

Dus drink ik me als een varken vol!

Kwam ik thuis zonder cent of sol,

haar scheldpartij verrok mijn pezen! 
[Ma (bonne) femme est morte, je suis ressuscité! / Alors je bois tout mon soûl comme un porc! / Si je rentrais sans un sou ou un sol, / sa bordée d'injures me tordait les nerfs.]

Les traits sont accusés: femme devient wijf, un régionalisme ici mais qui peut avoir un sens péjoratif; la différence entre «je suis libre!» et «je suis ressuscité!» est plus culturelle que religieuse; «boire tout mon soûl» donne «se remplir comme un cochon»; «ses cris», « une bordée d'injures»; et «me déchiraient la fibre» devient beaucoup plus concret avec «me tordaientt / détiraient les nerfs » où verrok représente une forme forte, régionale, du verbe régulier verrekken (se faire une élongation, une entorse), et le terme pezen, à l'image de «fibre», joue sur l'ambigüité entre nerfs et tendons.

Cette coloration n'est pas limitée aux thèmes populaires. À la fin de «Spleen. Je suis comme le roi d'un pays pluvieux», "l'eau verte du Léthé» est traduit par «Letheslijk» où slijk renvoie à la vase, la fange, y compris au sens moral. Le glissement culturel est un gain pour le poème comme le montre la traduction des derniers vers:

Il n'a su réchauffer ce cadavre hébété

Où coule au lieu de sang l'eau verte du Léthé. (Baudelaire 1975)

kon hij verwarmen niet dit afgestompte lijk, waardoor geen bloed meer vloeit maar 't groene Letheslijk.

[Il ne pouvait réchauffer ce cadavre hébété / à travers lequel ne coule plus de sang mais la vase verte du Léthé.]

Le premier vers présente une syntaxe inhabituelle où la négation niet est placée après l'infinitif pour marquer la césure. En évitant la construction avec «au lieu de», Decorte crée un vers ïambique au mouvement continu, tirant le meilleur parti des assonances et des allitérations et, surtout, concluant le poème par une rime riche à forte charge sémantique. L'avantage apparaît lorsque l'on compare avec les deux traductions de 1995, qui sont exactes (stroomt et vloeit sont des synonymes signifiant couler) et métriques, mais avec peu d'allant ("Waarin in plaats...») et dont la rime tombe sur des termes secondaires:

Traduction de Petrus Hoosemans (Baudelaire 1857, traduction 1995/1997):

Heeft nooit meer warmte in dit bot karkas gegloeid, waarin in plaats van bloed het groene Lethewater vloeit.

[Plus jamais la chaleur n'a dans cette grossière carcasse rayonné / dans laquelle au lieu de sang l'eau verte du Léthé coule.]

Traduction de Peter Verstegen (Baudelaire 1857, traduction 1995)

Heeft hij met nieuwe gloed dit bot karkas doorstroomd

Waarin, in plaats van bloed, het groene Lethewater stroomt.

[Il a d'une nouvelle énergie cette grossière carcasse irrigué / Dans laquelle, au lieu de sang, l'eau verte du Léthé coule.]

L'espace littéraire que la traduction de Decorte contribue à développer s'est constitué à partir du milieu du $\mathrm{XIX}^{\mathrm{e}}$ siècle. De la prise de conscience, à la fin du $\mathrm{XVIII}^{\mathrm{e}}$ siècle, du mépris dans lequel était tombé le néerlandais à la publication des premiers recueils de Guido Gezelle, entre 1858 et 1862, la littérature de Flandre appartient surtout aux philologues et aux historiens. Avec le prêtre-poète Gezelle, pour qui la gloire de Dieu, du flamand (occidental) et de la Flandre sont indissociables, 
la littérature devient un acteur essentiel. Se pose alors la question des relations avec les littératures voisines. L'attraction de la France est alors si puissante et trouve encore si peu de contrepoids que le sentiment flamand est d'abord porté par une succession d'écrivains francophones, de Camille Lemonnier (1844-1913) et Georges Eekhoud (1854-1927) à Georges Rodenbach (1855-1898), d'Émile Verhaeren (1855-1916) à Max Elskamp (1862-1931) et au prix Nobel de littérature Maurice Maeterlinck (1862-1949). Cette explosion créatrice est parfois vécue comme «un véritable pillage de la beauté, de l'art et de la poésie, et surtout de la gloire littéraire» (De Mont 1903: 300) ${ }^{12}$.

La réaction néerlandophone va se cristalliser autour de la revue Van Nu en Straks [D’aujourd'hui et de demain], créée en 1893. Pour Prosper van Langendonck (18621920) ou Karel van de Woestijne (1878-1929), Baudelaire n'est pas un auteur étranger, ni dans l'esprit ni par la langue. Ils reconnaissent son influence sur leur vision de l'homme et de la littérature, mais c'est une présence qui participe de leur formation francophone d'écrivain flamand. Un clivage intime se fait ainsi entre une inspiration venue de France et une expression littéraire flamande dans lequel l'œuvre baudelairienne, relayée par la revue La Jeune Belgique (1881-1897), joue un rôle pivot (Musschoot 1995). Elle permet en effet, par la thématisation poétique d'une division insurmontable de l'être, d'exprimer ce déchirement, puis de lui donner une forme flamande en néerlandais. L'objectif des auteurs réunis autour de Van Nu en Straks est de surmonter cette coupure intime en donnant naissance à une littérature réellement flamande et moderne.

Baudelaire et Rimbaud jouent ainsi, de manière paradoxale, un rôle dans le double effort de libération que mènent certains poètes flamands du pittoresque, lequel enferme la Flandre dans des stéréotypes, et d'une morale catholique, héritée de la contreréforme. C'est ce qu'August Vermeylen (1872-1945) résume en 1900 par une formule souvent reprise: "pour être quelque chose, nous devons être des Flamands. - Nous voulons être des Flamands pour devenir des Européens» (Vermeylen 1900: 310) ${ }^{13}$.

Les Fleurs du Mal donne à Decorte la possibilité d'explorer ses capacités créatrices. Le poète français fait de lui un poète davantage flamand. Ce paradoxe apparent tient aux rapports que la littérature en Flandre a tissés avec la littérature française, notamment par le truchement des mouvements d'avant-garde francophones bruxellois. Le traducteur est d'abord un lecteur formé par sa propre littérature. La traduction qu'il entreprend doit être placée dans le prolongement de cette lecture - et écriture - comme une expérience individuelle nécessairement déterminée, par opposition à une approche textuelle se proposant une manière de reconstruction. La liberté qui en résulte trouve notamment son expression dans les continuités que l'on découvre entre la traduction de Decorte et son œuvre, entre son œuvre et la situation de la littérature flamande. Sans doute pourrait-il se retrouver dans cette phrase de Clive Scott:

I have given text of my own the first and last word, to enact that being-in-the-text that translation is, to make Baudelaire's text an incident within my own discourse with myself, as a reader of Baudelaire, and as a translator. (Scott $2000: 220$ )

\section{Conclusion}

L'idée de création dans le domaine de la traduction est indissociable de l'infini des possibles qui s'offre au traducteur. Le traducteur flamand des Fleurs du Mal assume cette liberté et la justifie par la cohérence de ses choix et l'harmonie à la fois fidèle et 
neuve des poèmes composant De bloemen van den booze. Laccent mis sur la musicalité du vers, nécessairement transposée, oriente le processus de traduction et assure l'unité d'inspiration. La métrique est la structure dominante régissant la distribution des mots, et souvent leur forme, en fonction des positions stratégiques du vers. La rime y gagne en expressivité tandis que les tensions liées aux contraintes de la traduction se trouvent reportées au début du vers et après la césure, où le vers est moins structuré. C’est là que le rythme métrique, déterminé par les positions fortes, est le plus efficace pour régulariser le vers pour l'oreille.

La recherche d'équivalences métriques a montré un accord de fond entre les systèmes français et néerlandais en ce qui concerne les vers longs. La traduction des vers impairs en révèle cependant les profondes différences. Le nombre d'accents l'emporte sur le nombre de syllabes, entraînant une forte tendance à recourir au vers pair immédiatement supérieur.

Ces contraintes et ces solutions ne relèvent pas seulement de l'expérience individuelle du traducteur. Alors que les congrès néerlandais organisés à partir de 1849 œuvrent à un rapprochement linguistique entre Nord et Sud, les différences littéraires s'affirment et se creusent. La manière dont Decorte traduit Baudelaire est l'aboutissement d'un processus par lequel une littérature nouvelle s'est construite. Sa force créatrice résulte de l'accord entre la traduction, l'œuvre originale du poète-traducteur et leur insertion dans un contexte littéraire et culturel précis. De bloemen van den booze n'est pas une traduction isolée et demeure, de ce fait, un texte actif, suscitant de nouvelles expériences.

On sait que Baudelaire, fuyant les créanciers, déçu dans ses espoirs de gagner quelque argent et dont le corps cédait à la maladie, a pris en grippe cette Belgique bourgeoise, industrieuse et libérale, mais son regard reste acéré. À propos d'une possible annexion du pays, sujet débattu dans la presse française, Baudelaire écrit:

Razzia des richesses.

On peut déménager tout ce qui est beau. Chaque nation a le droit de dire: Cela m'appartient, puisque les Belges n'en jouissent pas. (Baudelaire 1976: 921) ${ }^{14}$

L'absence de jouissance, c'est l'absence d'une langue-littérature fondatrice. Aucune langue importée ne peut convenir. Ou alors, en effet, autant « déménager tout ce qui est beau», d'autres en feront un meilleur usage.

L'idée d'un Baudelaire flamand était à la fois absurde et incongrue. Soixante-dix ans plus tard seulement, une traduction en dessine les contours, renouvelant les rythmes et les images, puisant dans des strates de la langue où les souvenirs du maître français n'ont pas disparu. Ces strates préparent les traductions à venir, car, il faut y revenir, «il n'y a de jouissance poétique, bonheur esthétique de la traduction, que si la langue est consentante» (Ladmiral 2014: 25).

\section{NOTES}

* EA 4074 - CECILLE - Centre d’Études en Civilisations, Langues et Lettres Étrangères.

1. Texte original cité d'après:

Baudelaire, Charles (1975): Les Fleurs du Mal. In: Claude Pichois, dir. Euvres complètes. Vol. 1. Bibliothèque de la Pléiade. Paris: Gallimard.

Traductions citées dans cet article:

Baudelaire, Charles (1857/1914): Twee gedichten van Baudelaire vertaald door Albert Verwey [Deux poèmes traduits par A.V.]. (Traduit par Albert Verwey) De Beweging. 10:94-95. 
BAudelaire, Charles (1857/1919): Baudelaire: De bloemen van het kwade. Vertalingen door Albert Verwey [Baudelaire: Les fleurs du mal. Traductions par A.V]. (Traduit par Albert Verwey) De Beweging. 15:75-85,347-352.

Baudelaire, Charles (1857/1946): De bloemen van den booze [Les fleurs de l'alcool]. (Traduit par Bert Decorte) Anvers/Bussum: Orion/F.G. Kroonder.

Baudelaire, Charles (1857/1995): De bloemen van het kwaad [Les fleurs du mal]. (Traduit par Peter Verstegen) Amsterdam: G.A. van Oorschot.

Baudelaire, Charles (1857/1995/1997): De bloemen van het kwaad [Les fleurs du mal]. (Traduit par Petrus Hoosemans) $2^{\mathrm{e}}$ éd. Groningue: Historische Uitgeverij.

2. Ouvrages cités du traducteur:

Decorte, Bert (1935): De ruiters [Les cavaliers]. Forum. 4-6:510-513.

Decorte, Bert (1937/2012): Germinal. (Édité par Femke Vandevelde et Edward Vanhoutte) Gand: Koninklijke Academie voor Nederlandse Taal- en Letterkunde.

Decorte, Bert (1947): Aards gebedenboek [Bréviaire terrien]. Anvers: Orion.

Decorte, Bert (1971): Kortom [Bref]. Open Kaart. Bruges: Orion.

Decorte, Bert (1974): Verzamelde gedichten [CEuvre poétique]. De Gulden Veder. Bruges: Orion. Decorte, Bert (1984): De pijnlijke vreugde of het plezier van vertalen [La joie douloureuse ou le plaisir de traduire]. Verslagen en mededelingen van de Koninklijke Academie voor Nederlandse Taal- en Letterkunde. 1:362-371.

3. Vercamme, Jan (1936): Vlaamsche poëzie [Poésie flamande]. De Gemeenschap. 12:667-670.

4. Du Perron, Eddy (1935): Blocnote klein formaat [Blocnote de petit format]. Forum. 4-6:1208-1218.

5. Vermeylen, August (1953): De Vlaamse letteren van Gezelle tot heden [Les lettres flamandes de Gezelle à nos jours]. In: Herman Teirlinck et al., dir. Verzameld werk. Vol. 3. Bruxelles: Manteau, 573-757.

6. «[...] het eerste wonderkind in onze letterkunde sedert Van Ostaijen. Decorte bezit een overweldigende vitaliteit, die zich in onstuimige rhythmen beweegt.» DemedTs, André (1937) : De jongste Vlaamse literatuur [La littérature flamande la plus récente]. De Gemeenschap. 13:514-521.

7. Le lecteur francophone pourra se reporter au recueil suivant: GEZELLE, Guido (1999): Un compagnon pour toutes les saisons. (Traduit par Liliane Wouters) Marseille: Autres Temps.

8. Toutes les traductions littérales sont de l'auteur.

9. Notation: $\mathrm{w}=$ weak, syllabe non accentuée; $\mathrm{S}=$ strong, syllabe portant un accent.

10. «Deze was gesteld in een idioom dat niet kan worden beschouwd als gangbaar Nederlands. " Verstegen, Peter (7 juin 1995): De puberale branie van Michael Zeeman [Les fanfaronnades pubertaires de Michael Zeeman]. De Groene Amsterdammer. Consulté le 14 novembre 2015, www. groene.nl/artikel/de-puberale-branie-van-michael-zeeman.

11. Hietbrink (1999: 219) s'étonne que Verwey ait recours à l'hexamètre alexandrin pour traduire Baudelaire alors qu'il prône le pentamètre pour le sonnet. En réalité, Verwey ne procède ainsi que pour un quart des vers.

12. «[...] een roof van schoonheid, van kunst en poëzie, van littereere glorie bovenal.» DE Mont, Pol (1903) : Een woord over de Frans-Belgiese lyriek van na 1880 [Un mot sur la poésie lyrique francobelge d'après 1880]. Groot Nederland. 1:297-325.

13. «[...] om iets te zijn moeten wij Vlamingen zijn. - Wij willen Vlamingen zijn, om Europeërs te worden.» Vermeylen, August (1900): Vlaamsche en Europeesche beweging [Mouvement flamand et européen]. Van Nu en Straks. 4:299-310.

14. Les italiques sont de Charles Baudelaire. Baudelaire, Charles (1976): Pauvre Belgique! In: Claude Pichois, dir. Euvres complètes. Vol. 2. Bibliothèque de la Pléiade. Paris: Gallimard, 819-961.

\section{RÉFÉRENCES}

Boorj, Geert (1995): The Phonology of Dutch. The Phonology of the World's Languages. Oxford/ New York: Oxford University Press.

Brems, Elke (2012): De alcohol van Rimbaud. Decorte onder invloed [L'alcool de Rimbaud. Decorte sous influence]. In: Bert Decorte. Germinal. (Édité par Femke Vandevelde et Edward Vanhoutte) Gand: Koninklijke Academie voor Nederlandse Taal- en Letterkunde, 203-217.

De GeEst, Dirk (2012): De tucht van de ontucht? 'Germinal' van Bert Decorte als een poëticaal 'geval'. In: Bert Decorte. Germinal. (Édité par Femke Vandevelde et Edward VanHоUtтe) Gand: Koninklijke Academie voor Nederlandse Taal- en Letterkunde, 219-238. 
Erens, Frans (1906) : Literaire wandelingen [Promenades littéraires]. Amsterdam: S.L. van Looy, 46-61.

Gouvard, Jean-Michel (2000): Critique du vers. Métrique française et comparée. Paris: Honoré Champion.

Gouvard, Jean-Michel (2007): Métrique et variations dans Hier régnant désert d'Yves Bonnefoy. Semen. 24:28p. Consulté le 21 août 2016, http://semen.revues.org/5993?lang=en.

GrijP, Louis Peter (1992): De zingende Hadewijch. Op zoek naar de melodieën van haar Strofische Gedichten [La Hadewijch qui chante. À la recherche des mélodies de ses poèmes strophiques]. In: Frank Willaert, dir. Een zoet akkoord [Un doux accord]. Amsterdam: Prometheus, 72-92.

Halsema, Dick van (1995): Wie heel goed kijkt, die kan hem bijna zien [Qui regarde tres attentivement pourrait presque l'apercevoir]. In: Maarten van BUUREN, dir. Jullie gaven mij modder, ik heb er goud van gemaakt. Over Charles Baudelaire [Vous m'avez donné de la boue, j'en ai fait de l'or. À propos de Charles Baudelaire]. Groningue: Historische Uitgeverij, 66-110.

Hietbrink, Martin (1999): In de schaduw van Stefan George. De Baudelaire-vertalingen van Verwey [Dans l'ombre de S. G. Les traductions de Baudelaire par V.]. Tijdschrift voor Nederlandse Taal- en Letterkunde. 115(3):218-235.

Kinker, Johannes (1810): Proeve eener Hollandsche prosodia... [Essai de prosodie hollandaise...]. Werken der Hollandsche Maatschappij van fraaije kunsten en wetenschappen. Amsterdam: Johannes Allart.

Kooij, Jan et Oostendorp, Marc Van (2003): Fonologie. Een uitnodiging tot de klankleer van het Nederlands [Phonologie: une invitation à l'étude des sons du néerlandais]. Amsterdam: Amsterdam University Press.

Ladmiral, Jean-René (2014): Sourcier ou cibliste. Traductologiques. Paris: Les Belles Lettres.

MACRIs, Spiros (1988): Frans Erens, Charles Baudelaire. In: Gilbert van DE Louw, dir. Alluvions '88: Pays-Bas-Flandres: Littératures arts plastiques. Paris/Metz: Didier-Érudition/Université de Metz, 67-86.

MACRIs, Spiros (2016): Enjeux épistémologiques et politiques de la métrique. In: Claude BlanCKAERT, Jacqueline LÉON et Didier SAmaIn, dir. Modélisations et sciences humaines. Figurer, interpréter, simuler. Histoire des sciences humaines. Paris: L'Harmattan, 353-370.

Musschоот, Anne Marie (1995): Karel van de Woestijne, een baudelairiaanse dichter [Karel van de Woestijne, un poète baudelairien]. In: Maarten van BuUren, dir. Jullie gaven mij modder, ik heb er goud van gemaakt. Over Charles Baudelaire [Vous m'avez donné de la boue, j’en ai fait de l'or. À propos de Charles Baudelaire]. Groningue: Historische Uitgeverij, 111-130.

NaAijkens, Ton (2002): De slag om Shelley en andere essays over vertalen [La bataille de S. et autres essais sur la traduction]. Nijmegen: Vantilt, 177-200.

Neijt, Anneke et Schreuder, Robert (2007): Rhythm versus Analogy: Prosodic Form Variation in Dutch Compounds. Language and Speech. 50(4):533-566.

Sсотт, Clive (2000): Translating Baudelaire. Exeter: University of Exeter Press.

Verwey, Albert (1907): L’orientation de la littérature néerlandaise. Mercure de France. 229:33-42.

Verwey, Albert (1931): Ritme en metrum [Rythme et mètre]. Santpoort: C.A. Mees.

Zonneveld, Wim (1992): Van Afflighem en Chaucer: Het leven van Sinte Lutgart als jambisch gedicht [D’Afflighem à Chaucer: La vie de sainte Lutgard comme poème ïambique]. RuyghBewerp. Utrecht: Vakgroep Nederlands Rijksuniversiteit Utrecht. 